\title{
Viscous dissipative free convective flow from a vertical cone with heat generation/absorption, MHD in the presence of radiative non-uniform surface heat flux
}

Bapuji Pullepu and Rayampettai Munisamy Kannan

\begin{abstract}
The problem of combined effect of heat generation/absorption and thermal radiation on unsteady, laminar, natural convective movement with MHD, viscous dissipation over a vertical cone in the presence of variable heat flux is considered. The converted FDE's of the flow which is of partial natured, unsteady, united and non-linear are solved numerically subject to proper boundary conditions by Crank-Nicholson scheme which is an efficient, correct and absolutely stable FDM. The velocity and thermal profiles are obtained and analyze to expose the outcome of heat generation/absorption and thermal radiation at different values of the MHD, Prandtl numeral, viscous dissipation and the exponent in the power law difference of the surface heat flux. The local as well as average shear stress and heat transfer rate are accessible and analyzed. The present outcome is compared by available outcome in literature and is originate to exist in excellent conformity.
\end{abstract}

Mathematics Subject Classification (2010): 65M06, 76R10.

Keywords: Cone, finite difference method, heat generation/absorption, MHD, thermal radiation, viscous dissipation variable heat flux.

\section{Nomenclature:}

$\begin{array}{ll}F_{0}^{\prime \prime}(0) & - \text { Shear-Stress co-efficient in Ref: }[12] \\ G r_{L} & - \text { Grashof number } \\ a & - \text { Constant } \\ g & - \text { Rate of change of velocity due to gravity } \\ k & - \text { Thermal conductivity } \\ k * & - \text { Mean sink co-efficient } \\ L & - \text { Reference span } \\ M & - \text { Magnetic constraint }\end{array}$


$n-\quad$ Exponent in power law variation in surface temperature

$N u_{x} \quad-$ Local Nusselt number

$N u_{X} \quad-$ Dimensionless Local Nusselt numeral

$\overline{N u} \quad-\quad$ Dimensionless average Nusselt numeral

$\operatorname{Pr} \quad-\quad$ Prandtl number

$q_{w} \quad-\quad$ Variable heat flux per unit area

$R \quad-$ Non- dimensional local radius of the cone

$r \quad-$ Local radius of the cone

$T^{\prime} \quad-$ Temperature

$T-$ Non-dimensional temperature

$t^{\prime} \quad-$ Time

$t \quad-$ Non-dimensional time

$U-$ Non-dimensional velocity in X-direction

$u \quad-$ Velocity component in X-direction

$V \quad-$ Non-dimensional velocity in Y-direction

$v \quad-$ Rate component in y-direction

$X \quad-$ Non-dimensional spatial co-ordinate

$x \quad-\quad$ Spatial coefficient along cone generator

$Y \quad-\quad$ Non-dimensional spatial coefficient along the normal

- to the cone generator

$y \quad-\quad$ Spatial coefficient along the normal to the cone generator

\section{Greek Symbols:}

$\alpha \quad-\quad$ Thermal diffusivity

$\beta-$ Volumetric thermal expansion

$\sigma \quad-\quad$ Electrical conductivity

$\sigma * \quad-\quad$ Stefan-Boltzmann constant

$\Delta \quad-$ Non-dimensional heat source/sink constraint

$\Delta t \quad-$ Non-dimensional time step

$\Delta X-$ Non-dimensional finite difference grid size in $\mathrm{X}$-direction

$\Delta Y-$ Non-dimensional finite difference grid size in $\mathrm{Y}$-direction

$\epsilon \quad-\quad$ Viscous dissipation parameter

$\phi \quad-\quad$ Semi vertical angle of the cone

$\mu \quad-$ Dynamic viscosity

$\gamma-$ Kinematic viscosity

$\rho \quad-$ Density

$\tau_{x} \quad-\quad$ Local skin friction

$\tau_{X} \quad-$ Non-dimensional local skin friction

$\tau \quad-$ Non-dimensional average skin friction

\section{Subscripts:}

$w \quad-\quad$ Condition on the wall

$\infty \quad-\quad$ Free stream condition 


\section{Introduction}

Free convection boundary layer flow and thermal transport over a vertical cone has been the subject of attention of several investigators because these phenomenons arise regularly in environment, as good as in industrialized and technical applications. In the field of engineering, MHD has two areas of applications namely, Magneto hydrodynamic propulsion and power generation. MHD generator is based on the concept of using ionized gases as the moving conductor. The effect of thermal diffusion on the MHD free convection and mass transfer flow has important role in isotopes separation and in mixtures between gases. MHD flowing in an electrically conducting fluid are encountered in many industrial applications, such as purification of molten metals, non-metallic intrusion, liquid metal, plasma studies, geothermal energy extraction, nuclear reactor and the boundary layer control in the field of aerodynamics and aeronautic. Anjalidevi and Kandasamy [3] have analyzed the effects of chemical reaction, temperature and mass transport on non-linear MHD laminar boundary layer flow over a wedge among suction and injection. Analytical solution for the largely thermal and mass transport on MHD movement of an unvaryingly expanded vertical permeable surface with the effects of thermal source/sink and chemical response were obtainable by Chamkha [5].

Hakeem et al. [1] reported the methodical solutions for thermal and mass transport by laminar movement of an electrically performing fluid on a continuously vertical porous surface in the occurrence of a radiation and chemical response effect. Elbashbeshy et al. [10] investigated the laminar natural convection from a vertical circular cone with uneven surface heat flux in the existence of the pressure effort. As the difference between the surface temperature and the ambient temperature is large. The radiation upshots become vital. During the part of convection radiation, Viskanta and Grosh [25] considered the effect of temperature radiation on the thermal sharing and the thermal transport in an absorbing and emitting medium pouring in excess of a wedge by using the Rosseland diffusion estimate. These guesses leads to a significant generalization in the expression for the radiant flux. Muthucumarasamy and Ganesan [18] discussed heat effects on flow past an on impulsively established infinite vertical plate with uneven temperatures using the Laplace transform method.

Alam et al. [2] investigated the crisis of laminar free convective flow and thermal transport from a vertical permeable round cone retained at a non-uniform surface temperature with the force effort governed by the exponent law variation by the span from the convection boundary-layer flow of a micro-polar fluid above a vertical permeable cone with a changeable wall temperature. Cheng [9] analyzed a free convection boundary layer flow of a micro glacial fluid over a vertical permeable cone with a non-uniform surface temperature. Chen et al. [8] explored the free convection on parallel, inclined and vertical plates by dissimilar combination of non-uniform surface heat or changeable heat flux. Kabeir et al [11] used perturbation scheme to study the outcome of temperature and mass transport on natural convection flow with an unvarying suction and injection over a cone in a micro polar fluid. Makinde [14] analyzed hydromagnetic mixed convection flow and mass transport over a vertical porous plate by stable heat flux surrounded in a porous medium. Chamkha and Khaled [6] 
considered hydromagnetic combined heat and mass transport by free convection from a porous vertical plate embedded in a liquid saturated porous medium in the existence of temperature production or assimilation. Patra et al. [20] investigated the outcome of radiation on natural convection movement of a viscous and incompressible fluid close to a vertical flat plate with inclined temperature. They compared the effects of Radiative temperature transmit on free convection flow near a ramped temperature plate by the flow close to a uniform plate.

Mohamed et al. [15] investigated transient MHD natural convection temperature and mass transport boundary layer flow of viscous, incompressible, optically fat and electrically conducting fluid throughout a permeable medium along an on impulsively moving heat vertical plate in the existence of uniform chemical response of first order and heat reliant temperature drop. They obtained analytical answer of the leading equation in closed form by Laplace transform method. Mosa et al. [16] considered the Bouger numeral effects or glowing MHD Ekman flow on a permeable platter, present closed shape solution for together the optically lean and optically-thick case (achieve when Bouger numeral $>>1$, so that the mean free pathway of the emission is much lesser than the distinguishing measurement (i.e.) the scatter case) performance that for permanent magnetic field, the temperature distributions are powerfully affect by radiative fluctuation.

Beg et al. [4] focused the temperature source/sink effects on oscillatory magneto convection in a permeable medium using hypergeometrices. Chamkha et al. [7] considered combined temperature generation/absorption, emission and magnetic field effects on forced convection temperature transfer over a wedge with stress work effects. Muralidharan and Muthucumarasamy [17] Radiative heat transport effects on transient movement of viscous non-compressible fluid past an unvaryingly accelerated never-ending vertical plate by changeable heat and homogeneous mass flux have been investigated.

Sharma and Varshney [24] discussed the effects of temperature distribution and viscous dissipation on the transient flow of a viscous incompressible dirty gas throughout a hexagonal channel of regular cross-section under the influence of a magnetic field and moment-dependent pressure gradient.

\section{Mathematical analysis}

A 2-dimensional uniform, consisting of laminar free convection of thermal through a liquid or gas caused by molecular motion flow of a incapable of being compressed viscous fluid over a vertical cone with non-isothermal surface temperature under the determination of reaction due to the presence of electrically conducting and radiating liquid past a vertical cone with non-homogeneous heat flux by talking into an account the effects of viscous dissipation is viewed or the sensation caused by heat energy is carefully weighed with the following assumptions.

(i) The system is axi-symmetrical.

(ii) The Joule heating of the fluid (magnetic dissipation) is neglected.

(iii) The co-efficient of electrical conductivity is a stable throughout the fluid. 


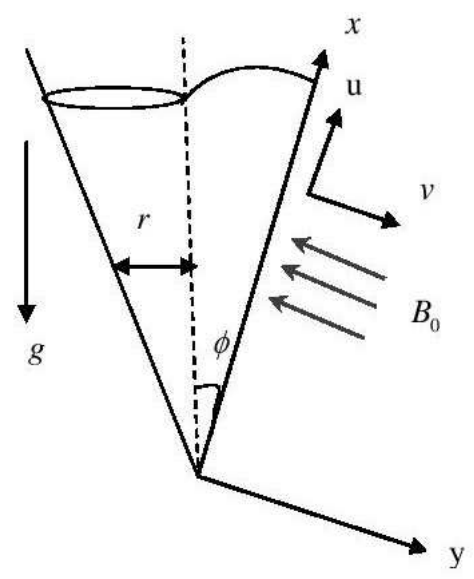

Figure 1. Physical Model and Coordinate System

(iv) Transverse uniform magnetic field is applied perpendicular to the surface of the cone.

(v) The attractive Reynolds number is little so that the induce magnetic field is neglect and consequently, do not alter the magnetic field.

(vi) Thermal radiation is current in the appearance a unidirectional flux in the $y$ direction

(vii) The Radiative thermal flux into the $\mathrm{x}$ direction is regard as unimportant in comparison with that in the $y$ direction.

(viii) The magnetic field equation is the common electromagnetic and hydro magnetic equation, but the communication between the flow and the attractive field is taken in to account.

(ix) Maxwell's dislocation current is ignored, so as to electric current is regard as flow in closed circuits.

The co-ordinate system is chosen such to establish position is give an exhibition of to an interested study has be consider as x-axis is in use on the surface of the cone from the vertex $x=0$ and y denotes the distance normally outward. The fluid belongings are taken to be unvarying and take exception to density divergences which stimulate perkiness strength term in the velocity equation and it maneuver key factor the discussion. Here $\phi$ is the half vertical angle of the cone and $r(x)$ is the local radius of the cone.

Primarily at $t^{\prime} \leq 0$, it is moreover considered that the cone surface and the enclosing fluid, which is at rest, have the same temperature $T_{\infty}^{\prime}$. Then at $t^{\prime}>0$, the temperature of cone surface is suddenly raised to inconsistently $q_{w}(x)=a x^{n}$. It is considered that the fluid properties are non-varying except for density variations, which induce buoyancy force term in the momentum equation. The governing boundary layer equations of continuity", an impelling force or strength and a thermodynamic 
quantity equivalent to the capacity of a physical system to do work which was proved by an approximation given by Boussinesq are stated below:

Equation of continuity:

$$
\frac{\partial}{\partial x}(r u)+\frac{\partial}{\partial y}(r v)=0
$$

Equation of momentum:

$$
\frac{\partial u}{\partial t^{\prime}}+u \frac{\partial u}{\partial x}+v \frac{\partial u}{\partial y}=g \beta \cos \phi\left(T^{\prime}-T_{\infty}^{\prime}\right)+\nu \frac{\partial^{2} u}{\partial y^{2}}-\frac{\sigma B_{0}^{2} u}{\rho}
$$

Equation of energy:

$$
\frac{\partial T^{\prime}}{\partial t^{\prime}}+u \frac{\partial T^{\prime}}{\partial x}+v \frac{\partial T^{\prime}}{\partial y}=\alpha \frac{\partial^{2} T^{\prime}}{\partial y^{2}}+\frac{\mu}{\rho C_{P}}\left(\frac{\partial u}{\partial y}\right)^{2}+\frac{Q_{0}}{\rho C_{P}}\left(T^{\prime}-T_{\infty}^{\prime}\right)-\frac{1}{\rho C_{P}} \frac{\partial q_{r}}{\partial y}
$$

The primary and boundary condition are prescribed as

$$
\begin{aligned}
& t^{\prime} \leq 0: u=0, v=0, T^{\prime}=T_{\infty}^{\prime} \text { for all } x \text { and } y \\
& \left.t^{\prime}>0: u=0, v=0, \frac{\partial T^{\prime}}{\partial y}=\frac{-q_{w}(x)}{k} \text { at } y=0\right\} \\
& u=0, T^{\prime}=T_{\infty}^{\prime} \text { at } x=0 \\
& u \rightarrow 0, T^{\prime} \rightarrow T_{\infty}^{\prime} \text { as } y \rightarrow \infty
\end{aligned}
$$

Using the Roseland estimate for radiation [23], Radiative heat flux is reduced.

$$
q_{r}=\frac{-4 \sigma^{*}}{3 k^{*}} \frac{\partial T^{\prime}}{\partial y}
$$

where $\sigma^{*}$ the Stefan-Boltzmann is stable and $k^{*}$ is the represent mean absorption coefficient. It must be prominent that by using the Roseland approximation, the current study is restricted to optically substantial fluid. If temperature difference within the flow is adequately small, then equation (2.5) can be linearized by expanding $T^{\prime 4}$ in Taylor series about $T_{\infty}^{\prime}$ which after forgetting upper order conditions take the form:

$$
T^{\prime 4} \cong 4 T_{\infty}^{\prime 3} T^{\prime}-3 T_{\infty}^{\prime 4}
$$

Using equations (2.5) and (2.6), the energy equation (2.3) becomes

$$
\frac{\partial T^{\prime}}{\partial t^{\prime}}+u \frac{\partial T^{\prime}}{\partial x}+v \frac{\partial T^{\prime}}{\partial y}=\alpha \frac{\partial^{2} T^{\prime}}{\partial y^{2}}+\frac{\mu}{\rho C_{P}}\left(\frac{\partial u}{\partial y}\right)^{2}+\frac{Q_{0}}{\rho C_{P}}\left(T^{\prime}-T_{\infty}^{\prime}\right)+\frac{1}{\rho C_{P}} \frac{16 \sigma^{*} T_{\infty}^{\prime 3}}{3 k^{*}} \frac{\partial^{2} T^{\prime}}{\partial y^{2}}
$$

Local skin-friction and local Nusselt number has been given by

$$
\tau_{x}^{\prime}=\mu\left(\frac{\partial u}{\partial y}\right)_{y=0} ; N u_{x}^{\prime}=\frac{-x\left(\frac{\partial T^{\prime}}{\partial y}\right)_{y=0}}{T_{w}^{\prime}-T_{\infty}^{\prime}}
$$


Further, we introducing the subsequent dimensionless quantities:

$$
\left.\begin{array}{l}
X=\frac{x}{L}, Y=\frac{y}{L}\left(G r_{L}\right)^{\frac{1}{5}}, R=\frac{r}{L} \text { where } r=x \sin \phi \\
t=\frac{\nu t^{\prime}}{L^{2}}\left(G r_{L}\right)^{\frac{2}{5}}, T=\frac{T^{\prime}-T_{\infty}^{\prime}}{\frac{q_{w}(L)}{k^{* *}}}\left(G r_{L}\right)^{\frac{1}{5}}, \varepsilon=\frac{g \beta L}{C_{P}}, \\
U=\frac{u L}{\nu}\left(G r_{L}\right)^{\frac{-2}{5}}, V=\frac{v L}{\nu}\left(G r_{L}\right)^{\frac{-1}{5}}, \\
\gamma=\frac{\mu}{\rho}, \Delta=\frac{Q_{0} L^{2}}{C_{P} \mu}\left(G r_{L}\right)^{\frac{-1}{5}}, P r=\frac{\nu}{\alpha}, \\
M=\frac{\sigma B_{0}^{2} L^{2}}{\mu}\left(G r_{L}\right)^{\frac{-2}{5}}, R_{d}=\frac{k^{*} k}{4 \sigma^{*} T_{\infty}^{\prime 3}}, \\
G r_{L}=\frac{g \beta \frac{q_{w}(L)}{k^{* *}} L^{4} \cos \phi}{\nu^{2}}
\end{array}\right\}
$$

Equations (2.1) to $(2.3)$ can be written in the subsequent dimensionless form:

$$
\begin{gathered}
\frac{\partial}{\partial X}(U R)+\frac{\partial}{\partial Y}(V R)=0 \\
\frac{\partial U}{\partial t}+U \frac{\partial U}{\partial X}+V \frac{\partial U}{\partial Y}=T-M U+\frac{\partial^{2} U}{\partial Y^{2}} \\
\frac{\partial T}{\partial t}+U \frac{\partial T}{\partial X}+V \frac{\partial T}{\partial Y}=\frac{1}{\operatorname{Pr}}\left(1+\frac{4}{3 R_{d}}\right) \frac{\partial^{2} T}{\partial Y^{2}}+\Delta T+\varepsilon\left(\frac{\partial U}{\partial Y}\right)^{2}
\end{gathered}
$$

The following dimensionless primary and boundary condition are

$$
\left.\begin{array}{l}
t \leq 0: U=0, V=0, T=0 \text { for all } X \text { and } Y \\
t>0: U=0, V=0, \frac{\partial T}{\partial Y}=-X^{n} \text { at } Y=0 \\
U=0, T=0 \text { at } X=0 \\
U \rightarrow 0, T \rightarrow 0 \text { as } Y \rightarrow \infty
\end{array}\right\}
$$

Shear stress and heat transfer rate in dimensionless form are given by

$$
\begin{gathered}
\tau_{X}=\left(G r_{L}\right)^{\frac{3}{5}}\left(\frac{\partial U}{\partial Y}\right)_{Y=0} \\
N u_{X}=\frac{X}{T_{Y=0}}\left(\frac{-\partial T}{\partial Y}\right)_{Y=0}\left(G r_{L}\right)^{\frac{1}{5}}
\end{gathered}
$$

Also, the dimensionless average shear stress $\bar{\tau}$ and the average heat transfer rate $\overline{N u}$ are able to write as

$$
\begin{gathered}
\bar{\tau}=2 G r_{L}^{\frac{3}{5}} \int_{0}^{1} X\left(\frac{\partial U}{\partial Y}\right)_{Y=0} d X \\
\overline{N u}=2 G r_{L}^{\frac{1}{5}} \int_{0}^{1} \frac{X}{T_{Y=0}}\left(\frac{-\partial T}{\partial Y}\right)_{Y=0} d X
\end{gathered}
$$




\section{Method of solution}

The transient, non-linear, coupled PDE (2.10) to (2.12) with (2.13) are worked out by using Crank-Nicholson method. After applying the method the dimensionless equation converted to the system of tri-diagonal equations. We work out the scheme of equations by use well known Thomas algorithm by which we attain the desired solution with convergence of this algorithm occurring in a brief period of time and also it is unconditionally stable to change as discussed Bapuji et al. [22]. The integral area is treated as a rectangle with $X_{\max }=1$ and $Y_{\max }=26$ (the value for $Y$ is taken to be $\infty$ ) by analyzing in detail and considered in order to satisfy the ultimate and penultimate conditions of (2.13) and we observed that it is fulfilled with accuracy up to $10^{-5}$.

The mesh sizes has been permanent as $\Delta X=0.05, \Delta Y=0.05$ with time step $\Delta t=$ 0.01. The computations are carried out first by reducing the spatial mesh sizes by $50 \%$ in one way, and afterward in both direction by $50 \%$. The computations are conceded out first by reducing the spatial net sizes by $50 \%$. The results are compared. It is revealed that in all cases, the outcomes differ simply in the fifth decimal place. Hence, the choice of the mesh sizes seem to be suitable.

The co-efficient of $U_{i, j}^{k}$ and $V_{i, j}^{k}$ appearing in the FDE's are treated as constants at any one-time step. Here $\mathrm{i}$ designates the grid point along the $\mathrm{X}$ direction, $\mathrm{j}$ along the $\mathrm{Y}$ direction and $\mathrm{k}$ along the time t. The values of $\mathrm{V}, \mathrm{U}$ and $\mathrm{T}$ are known at all grid points when $t=0$ from the initial conditions.

The computations of $\mathrm{U}, \mathrm{V}$ and $\mathrm{T}$ at a time level $(k+1)$, using the values at previous time level $\mathrm{k}$ are carried out as follows. The FDE (2.12) at every internal nodal point on a particular i - level constitutes a tri-diagonal scheme of equations and is solved by Thomas algorithm as discussed in Bapuji et al [22]. Thus, the values of $\mathrm{T}$ are found out at every lattice point at a particular i at $(\mathrm{k}+1)$ th time level. Similarly, the values of $\mathrm{U}$ are calculated from equation (2.11), and finally the values of $\mathrm{V}$ are calculated explicitly by using equation (2.10) at every mesh point on a particular ilevel at $(\mathrm{k}+1)$ th time level. In a similar, manner computations are carried out by moving along i direction. Subsequent to calculating values corresponding to each i at a time level, the values at the next time level are determined in a similar manner. Computations are repeated until steady state is reached. The steady state solution is assumed to have been reached when the absolute difference between the values of the rate $\mathrm{U}$, as well as temperature $\mathrm{T}$ at two successive time steps are less than $10^{-5}$ at all grid points.

\section{Results and discussions}

This segment provides the behavior of a range of parameter involved in the expressions velocity and temperature the near result in stable condition at $X=1.0$ is established with accessible resemblance solution in literature. The momentum and thermal boundary layer profile of the cone with isothermal surface heat flux when $\operatorname{Pr}=0.72, R d=2.0, M=2.0$ and surface temperature power law exponent $n=0.5$ the numerical values of shear stress $\tau_{X}$ and rate of heat transfer $N u_{x}$ for distinct value 
of Prandtl number revealed in table (1) are examined with resemblance solution of Lin [13] in steady state using a appropriate conversion.

(i.e.) $Y=(20 / 9)^{1 / 5} \eta, T=(20 / 9)^{1 / 5}[-\theta(0)], U=(20 / 9)^{1 / 5} f^{\prime}(\eta)$, $\tau_{X}=(20 / 9) f^{\prime \prime}(0)$.

In adding up, the local shear stress $\tau_{X}$ and rate of heat transfer $N u_{x}$, for distinct numerical quantities of $\operatorname{Pr}$ when heat flux gradient $n=0.50$ and $M=0$ at $X=1.00$ instable situation are compared with the non-similarity result of Hossain and Paul [12] in table 2. It is noticed that the results are in good agreement with each other. It is also noticed that the current result concur well with those of Pop and Watanable [21], Na and Chiou [19] (as pointed out in table 1).

The transitory velocity and temperature profile at $X=1.0$ for dissimilar values of $\operatorname{Pr}$ and $M$ be plot in figs. 2 and 3. The viscous force increases and the thermal diffusivity decrease by a rising $P r$, which cause a decline in the velocity and temperature. Also, the influence of magnetic constraint $M$ against span-wise spatial distribution of velocity and temperature are depicted. Application of attractive meadow usual to the flow of an electrically conduct fluid give increase to an opposite force. These opposite powers tend to slow downward the movement of the fluid along the cone and cause a raise in its hotness and a decline in velocity as $M$ increase. An increases in $M$ from 1 although 2, 3 obviously reduce flow-wise velocity together in the close to-wall area and distant-field regime of the boundary layer, while the surface temperature increase as the bigger values of $M$.

Table 1: Relationship of steady - state shear stress and temperature values at $X=1.0$ with those of Lin [13] for isothermal surface heat flux.

* Values taken from Pop and Watanabe [21] when suction/injection is zero. ** Values taken from $\mathrm{Na}$ and Chiou [19] when solutions for flow over a full cone.

\begin{tabular}{|c|c|l|l|l|c|l|}
\hline \multirow{3}{*}{$\operatorname{Pr}$} & \multicolumn{3}{|c|}{ Temperature } & \multicolumn{3}{c|}{ Local skin friction } \\
\cline { 2 - 7 } & \multicolumn{2}{|c|}{ Lin [13] results } & $\begin{array}{l}\text { Present } \\
\text { values }\end{array}$ & \multicolumn{2}{c|}{ Lin [13] results } & $\begin{array}{l}\text { Present } \\
\text { values }\end{array}$ \\
\cline { 2 - 7 } & $-\theta(0)$ & $-\left(\frac{20}{9}\right)^{1 / 5} \theta(0)$ & $\mathrm{T}$ & $-f^{\prime \prime}(0)$ & $\left(\frac{20}{9}\right)^{2 / 5} f^{\prime \prime}(0)$ & $T$ \\
\hline 0.72 & 1.522878 & 1.7864 & 1.7714 & $\begin{array}{l}0.88930, \\
0.88930 *\end{array}$ & 1.224 & 1.2105 \\
\hline 1 & 1.39174 & $\begin{array}{l}1.6327, \\
1.6329 * *\end{array}$ & 1.6182 & 0.78446 & 1.0797 & 1.0669 \\
\hline 2 & 1.16209 & 1.3633 & 1.3499 & 0.60252 & 0.8293 & 0.8182 \\
\hline 4 & 0.98095 & 1.1508 & 1.1385 & 0.46307 & 0.6373 & 0.6275 \\
\hline 6 & 0.89195 & 1.0464 & 1.0344 & 0.39688 & 0.5462 & 0.5371 \\
\hline 8 & 0.83497 & 0.9796 & 0.9677 & 0.35563 & 0.4895 & 0.4808 \\
\hline 10 & 0.79388 & 0.9314 & 0.9196 & 0.32655 & 0.4494 & 0.4411 \\
\hline 100 & 0.48372 & 0.5675 & 0.5531 & 0.13371 & 0.184 & 0.1778 \\
\hline
\end{tabular}


Table 2: Comparison of steady-state shear stress and heat transfer rate values at $X=1.0$ with those of Hossain and Paul [12] for different values of $\operatorname{Pr}$ when $n=0.5$ and $M=0$ suction is zero.

\begin{tabular}{|c|c|c|c|l|}
\hline \multirow{3}{*}{$\operatorname{Pr}$} & \multicolumn{2}{|c|}{ Temperature } & \multicolumn{2}{c|}{ Local Nusselt number } \\
\cline { 2 - 5 } & Hossain and Paul [12] & $\begin{array}{l}\text { Present } \\
\text { values }\end{array}$ & Hossain and Paul [12] & $\begin{array}{l}\text { Present } \\
\text { values }\end{array}$ \\
\cline { 2 - 5 } & $F_{0}^{\prime \prime}(0)$ & $\frac{\tau_{X}}{G r_{L}^{3 / 5}}$ & $\frac{1}{\phi_{0}(0)}$ & $\frac{N u_{X}}{G r_{L}^{1 / 5}}$ \\
\hline 0.01 & 5.1345 & 5.1155 & 0.14633 & 0.1458 \\
\hline 0.05 & 2.93993 & 2.9297 & 0.26212 & 0.2630 \\
\hline 0.1 & 2.29051 & 2.2838 & 0.33174 & 0.3324 \\
\hline
\end{tabular}

Figs. 4 and 5 shows the effect of different heat generation/absorption parameter $\Delta$ and radiation constraint $\mathrm{Rd}$ on the dimensionless velocity and dimensionless thermal for the Prandtl number $\operatorname{Pr}=0.72, n=0.5, M=2$ and $\varepsilon=0.5$. It is revealed that the velocity and temperature profile are increase with rising quantities of $\Delta$. In raising $R d$ cause a considerable decreases in velocity with detachment into the boundary layer (i.e.) decelerate the flow. We too note down that with growing values of $R d$ the time taken to achieve the stable state is abridged. As estimated temperature value are also considerably abridged with increases in $R d$.

Figs. 6 and 7 represents the velocity and temperature profiles for dissimilar values of $n$ and viscous dissipative constraint $\varepsilon$. It is seen that the impulsive forces are decreased by the side of the cone surface close to the apex with increases in ' $n$ '. Owing to this, the dissimilarity involving the sequential utmost and stable state quantities decrease, the velocity with temperature decreases, the time requisite for the achievement of a stable state increase and velocity and the temperature boundary layer turn out to be thinner at lower values of $n$.

An increase viscous dissipative thermal cause an increase in the temperature, so as to the momentum boundary layer and thermal boundary layer increases with $\varepsilon$. It is as well see to facilitate the variation connecting the temporal utmost and stable state values are abridged, even as the moment necessary for the achievement of a steady state as well as the velocity and thermal boundary layer increases by $\varepsilon$.

Once, if temperature and velocity are found, it is interest to study local as well as average shear stress and heat transfer rate distributions in transitory state. The derivative in the equations $(2.14)$ - (2.17) is acquired with the use of five-point approximation formula and the integrals be calculated via the Newton-Cotes closed integration method. Figures 8 and 9 illustrate local Nusselt number and shear stress for various values of $\mathrm{Pr}$ and $M$. It is demonstrates that both quantity increases with decreasing $\mathrm{Pr}$ and decrease with distance from the cone vertex. An increasing magnetic parameter $M$ leads to decreasing both local shear stress and local heat transfer rate. Figs. 10 and 11 indicates the outcome of heat absorption/ generation constraint $\Delta$ and radiation constraint $R d$. The local shear stress increases for the higher values of $\Delta$. But the trend is reversed the heat transfer rate case. Also, stronger thermal radiations accelerate the flow but reduce Nusselt number hence the local skinfriction got decreased due to the presence of radiation. Consequently, heat transfer 
rate increases for lower value of $R d$. Figs. 12 and 13 depicts the effect of viscous dissipative constraint and the exponent in the power rule difference $\mathrm{n}$ on the local shear stress coefficient and Nusselt number are analyzed. From these graphs, it is able to be seeing that increasing $\varepsilon$ clearly boosts the wall shear stresses $\tau_{X}$, which grow powerfully from the directing boundary downstream alongside the cone surface.

Consequently, it enhances the viscous dissipative heats direct to a decline in the local heat transfer rate $N u_{X}$ (i.e.) with a substantial increase in $\varepsilon$ a strong reduce in the surface heat transfer rate. In figs. 14 and 15 shows the influence of Prandtl numeral and magnetic parameter over the average local skin friction and average heat transfer rate are observed. The average shear stress decreases for smaller value of $\mathrm{Pr}$ and larger value of magnetic parameter $M$. Also, it is noticed that the average heat transfer rate decreases for increasing values of Prandtl numeral $\operatorname{Pr}$ and $M$.

Figs. 16 and 17 demonstrates the results of heat source/sink constraint and radiation parameter $R d$. Stronger thermal radiation accelerate the flow but reduces average Nusselt number hence the average skin-friction got decreased due to the existence of magnetic field and radiation where as it boost for the higher values of thermal absorption/ generation constraint $\Delta$.

In figs. 18 and 19 depicts the variations of average shear stress and average heat transfer rate for controlling parameter $n$ and viscous dissipation parameter $\varepsilon$. Average shear stress is more for lower values of $n$ and average heat transfer rate is almost negligible. Also, it boost in $\varepsilon$ the viscous dissipative heat leads to boost in the average skin-friction. But the trend is reversed in average Nusselt number cases.

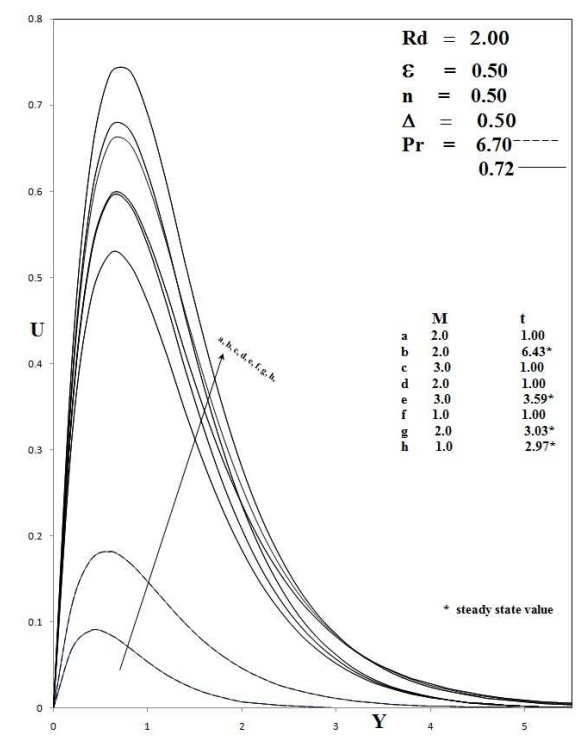

Figure 2. Transient velocity profiles at $X=1.0$ for different values of $\operatorname{Pr}$ and $M$ 


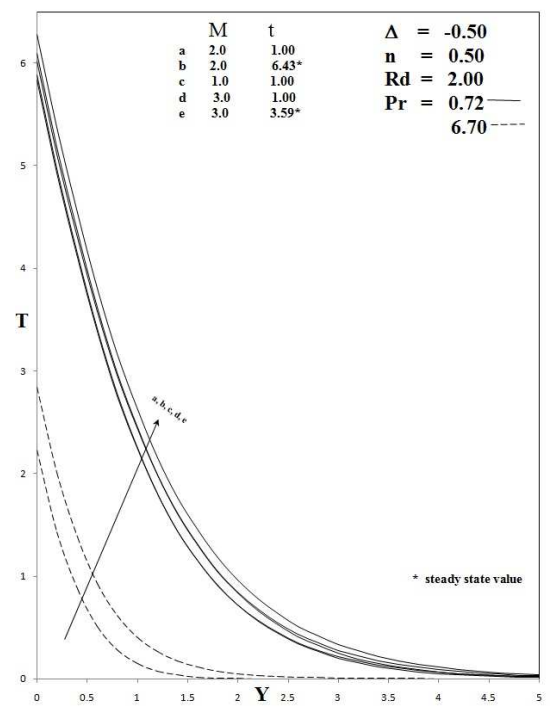

Figure 3. Transient temperature profiles at $X=1.0$ for different values of $\operatorname{Pr}$ and $M$

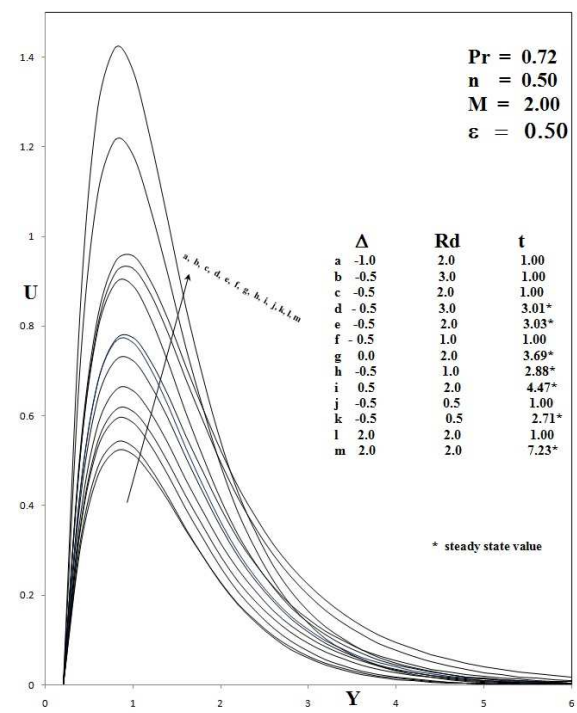

Figure 4. Transient velocity profiles at $X=1.0$ for different values of $\Delta$ and $R d$ 


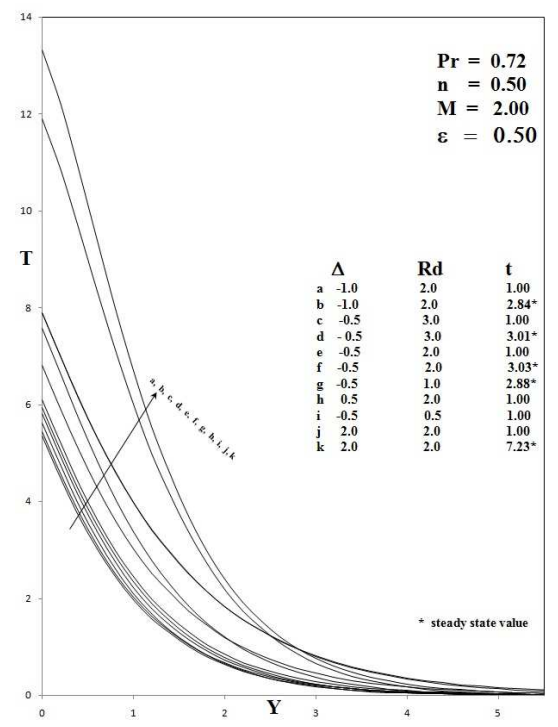

Figure 5. Transient temperature profiles at $X=1.0$ for different values of $\Delta$ and $R d$

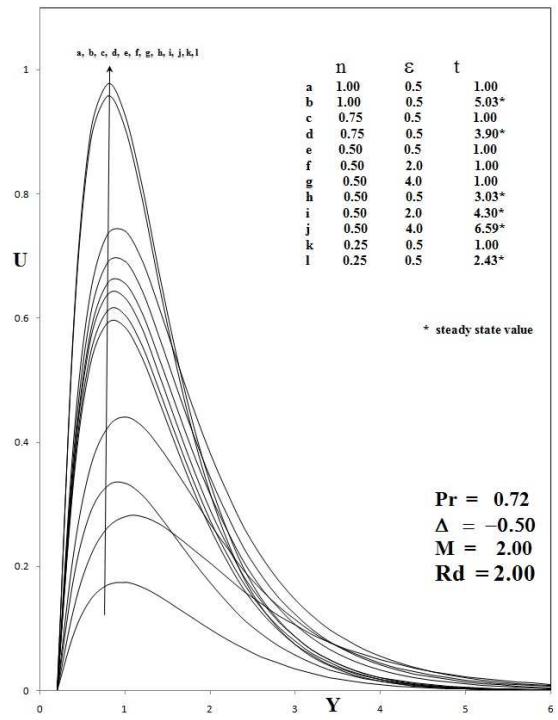

Figure 6 . Transient velocity profiles at $X=1.0$ for different values of $n$ and $\varepsilon$ 


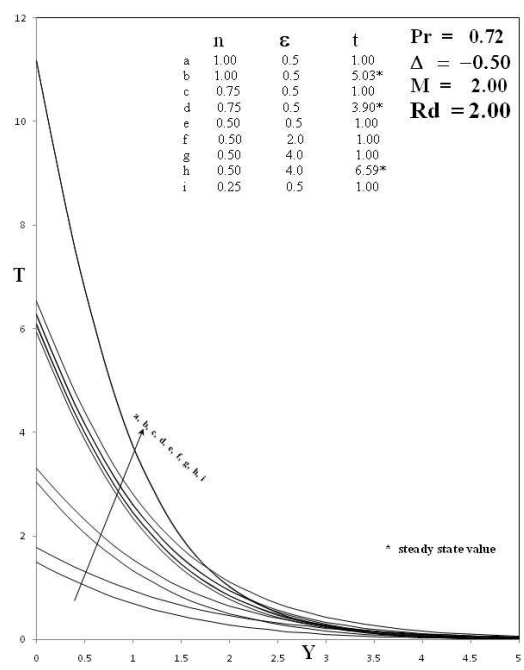

Figure 7 . Transient velocity profiles at $X=1.0$ for different values of $n$ and $\varepsilon$



FiguRe 8. Local skin friction for different values of $\operatorname{Pr}$ and $M$ in transient period 


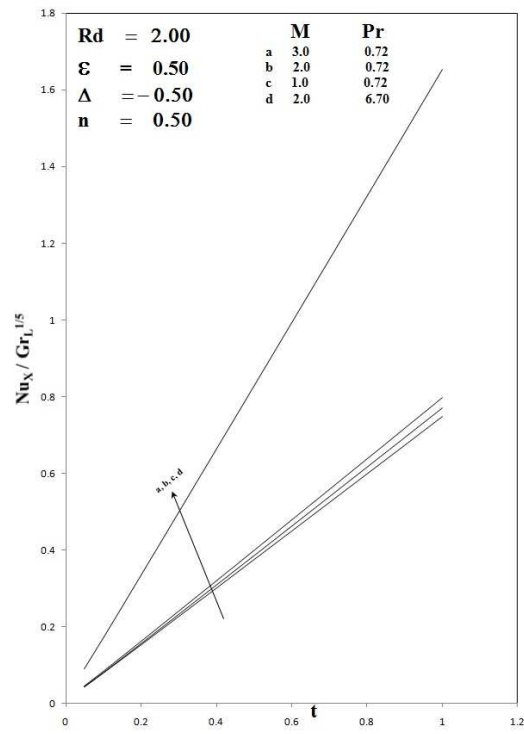

Figure 9. Local Nusselt number for different values of $\operatorname{Pr}$ and $M$ in transient period



Figure 10. Local skin friction for different values of $\Delta$ and $R d$ in transient period 


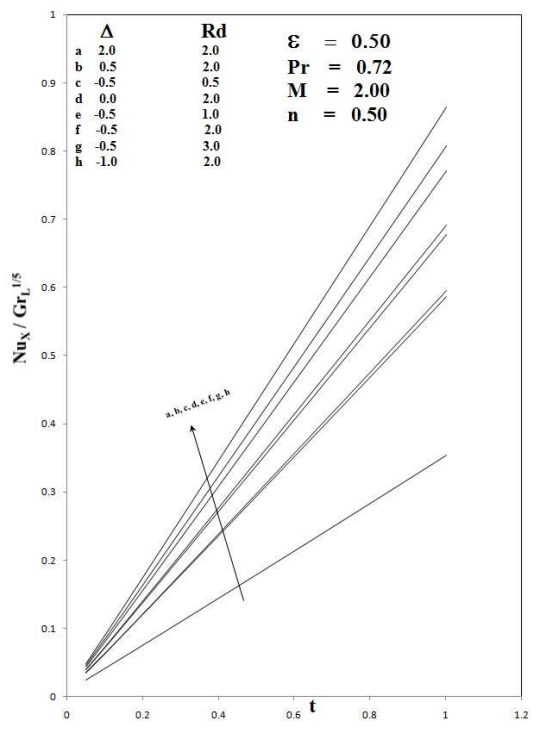

Figure 11. Local Nusselt number for different values of $\Delta$ and $R d$ in transient period

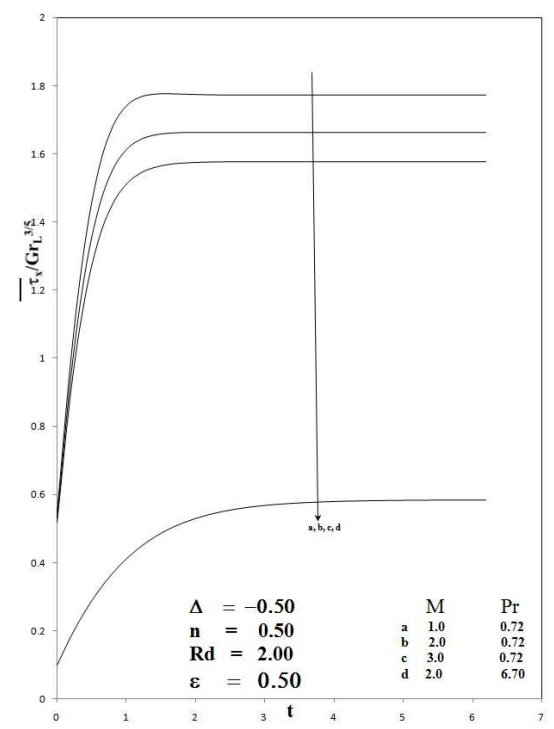

Figure 12. Average skin friction for different values of $\operatorname{Pr}$ and $M$ in transient period 


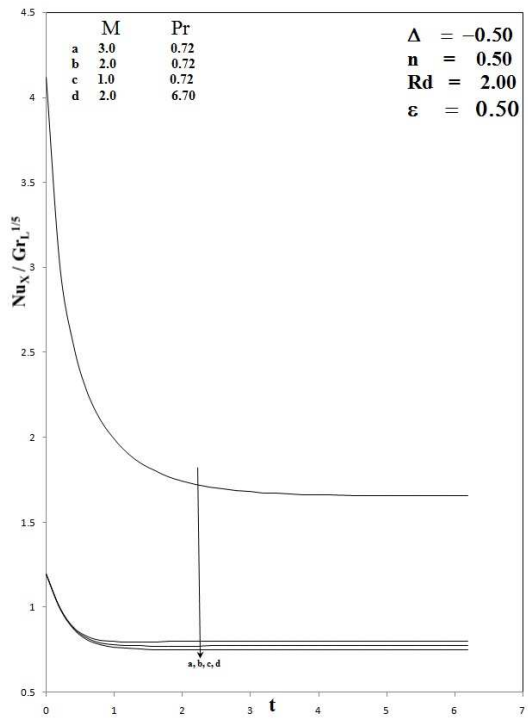

Figure 13. Average Nusselt Number for different values of $\operatorname{Pr}$ and $M$ in transient period



Figure 14. Average skin friction for different values of $\Delta$ and $R d$ in transient period 


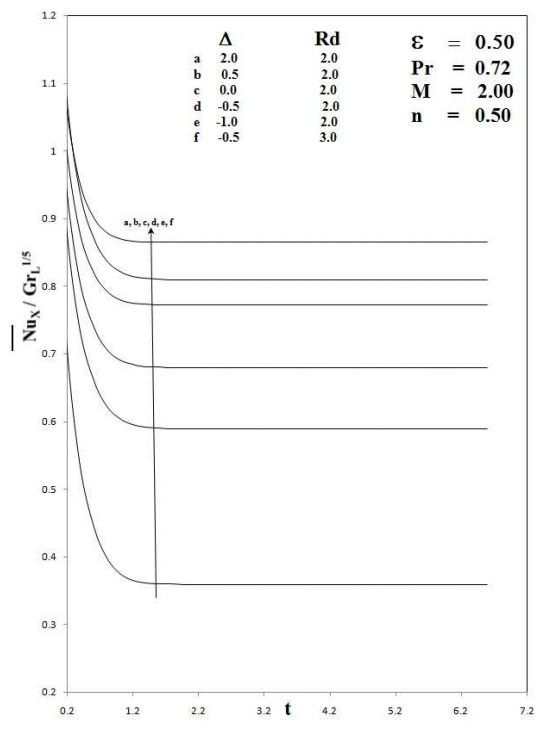

Figure 15. Average Nusselt number for different values of $\Delta$ and $R d$ in transient period



Figure 16. Average skin friction for different values of $n$ and $\varepsilon$ in transient period 


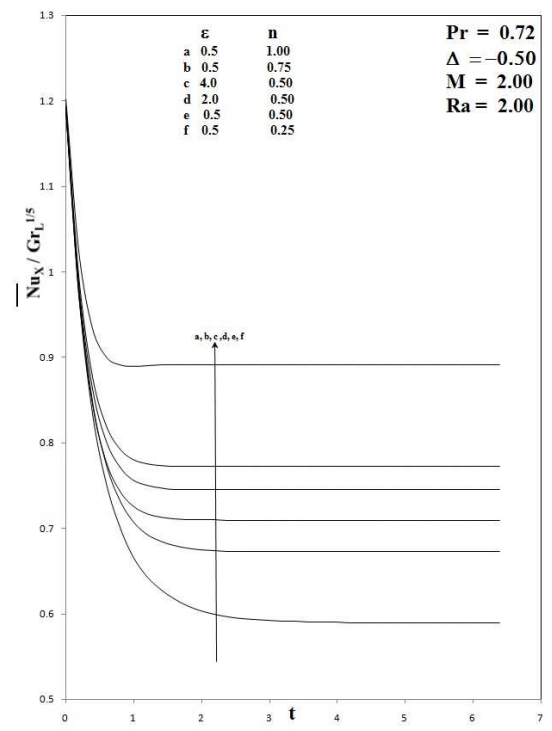

FiguRE 17. Average Nusselt number for different values of $n$ and $\varepsilon$ in transient period

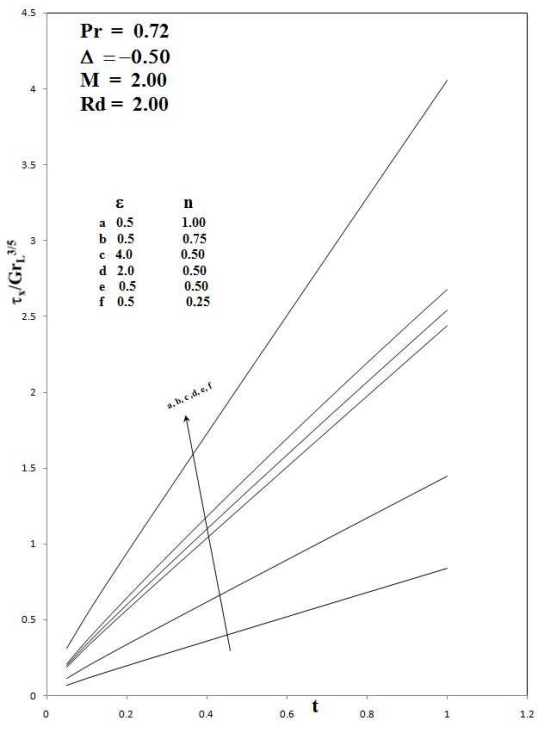

Figure 18. Local skin friction for different values of $n$ and $\varepsilon$ in transient period 


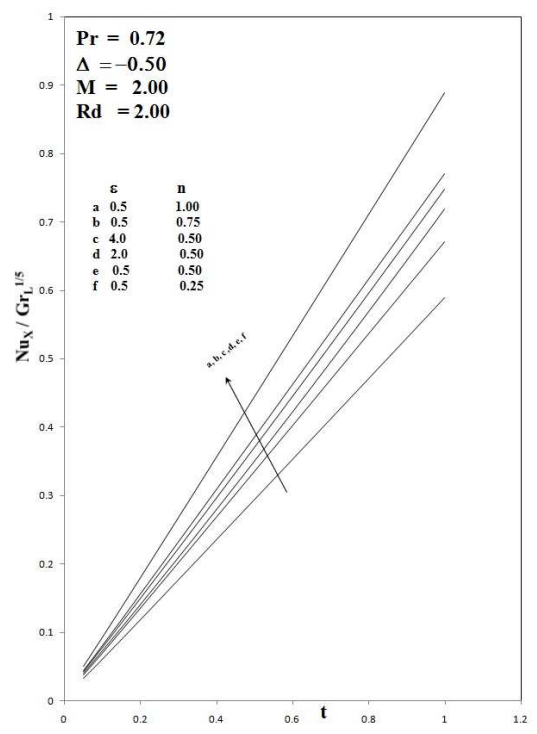

Figure 19. Local Nusselt number for different values of $n$ and $\varepsilon$ in transient period

\section{Conclusion}

A mathematical study of the flow past a variable vertical cone has been studied. The family of leading partial differential equations are solved via an implicit finite difference scheme of Crank-Nicholson type. The subsequent conclusions are drawn:

- The computations have shown that the velocity and temperature allocation decrease with growing the values of $\operatorname{Pr}, n$ and while the velocity and temperature allocation increase with increasing the value of $\Delta, \varepsilon$.

- The velocity increases and temperature decreases when the controlling parameter $\mathrm{M}$ and radiation parameter $R d$.

- The shear stress $\tau_{X}$ and heat transfer rate $N u_{X}$ values decrease as $M, n, R d$ and $\operatorname{Pr}$ increases.

- Smaller values of heat generation /absorption parameter $\Delta$ lead to decline in the values of the shear stress coefficient while the local Nusselt number increases.

- The average shear stress is more for larger values of $\Delta, \varepsilon$ and smaller values of $\operatorname{Pr}, n, R d$ and $M$.

- The average heat transfer rate increases for bigger value of $\operatorname{Pr}, n$ and $\varepsilon$ for lesser value of $\Delta, R d$ and $M$. 


\section{References}

[1] Abdul Hakeem, A.K, Muthukumar, S., Sathyanathan, K., Ganga, B., Magneto convective heat and mass transfer over a porous plate with effects of chemical reaction, radiation absorption and variable viscosity, International Journal of Energy and Technology, 2(2010), no. 23, 1-9.

[2] Alam, Md. M., Alim, M.A., Chowdhury, Md. M.K., Free convection from a vertical permeable circular cone with pressure work and non-uniform surface temperature, Nonlinear. Anal Modelg and Cont., 12(2007), no. 1, 21-32.

[3] Anjalidevi, S.P., Kandasamy, R., Effects of chemical reaction heat and mass transfer on non-linear MHD laminar boundary layer flow over a wedge with suction or injection, International Communication Heat Mass Transfer., 29(2002), 707-716.

[4] Anwar Beg, O., Singh, A.K., Takhar, H.S., Multi-parameter perturbation analysis of hydromagnetic oscillatory sources, Int. J. Fluid Mech., Res., 34(2006), 635-661.

[5] Chamkha, A.J., MHD flow of a uniformly stretched vertical permeable surface in the presence of heat generation/absorption and a chemical reaction, International Communication Heat Mass Transfer., 30(2003), 413-422.

[6] Chamkha, A.J., Khaled, A.A., Hydromagnetic combined heat and mass transfer by natural convection from a permeable surface embedded in a fluid saturated porous medium, Int. J. Numerical Method Heat Fluid Flow., 10(2000), 455-477.

[7] Chamkha, A.J., Quadri, M.A., Issa, C., Thermal radiation effects on MHD forced convection flow adjacent to a non-isothermal wedge in the presence of a heat source or sink, Heat mass Transfer., 39(2003), 305-312.

[8] Chen, T.S., Tien, H.C., Armaly, B.F., Natural convection on horizontal inclined and vertical plates with variable surface temperature or heat flux, International Journal of Heat Mass Transfer., 29(1986), 1465-1478.

[9] Cheng, C.Y., Natural convection boundary layer flow of a micro polar fluid over a vertical permeable cone with variable wall temperature, Int. Commun. In Heat and Mass Transfer., 38(2011), 429-433.

[10] Elbashbeshy, E.M.A, Emam T.G., Sayed E.A., Effect of pressure work on free convection flow from a vertical circular cone with variable surface heat flux, Strojnicky Casopis., 63(2012), no. 3, 169-177.

[11] El-Kabeir, S.M.M., Modather, M., Mansour, M.A., Effect of heat and mass transfer on free convection flow over a cone with uniform suction or injection in micro polar fluids, International Journal of Applied Mechanics and Engineering, 11(2006), no. 1, 15-35.

[12] Hossain, M.A., Paul, S.C., Free convection from a vertical permeable circular cone with non-uniform surface heat flux, Heat and Mass Transfer, 37(2001), 167-173.

[13] Lin, F.N., Laminar free convection from a vertical cone with uniform surface heat flux, Letters in Heat and Mass Transfer., 3(1976), 49-58.

[14] Makinde, O.D., On MHD boundary layer flow and mass transfer past a vertical plate in a porous medium with constant heat flux, Int. J. Numerical Method Heat Fluid Flow, 19(2009), 546-554.

[15] Mohamed, R.A., Osman, A.N.A., Abo-Dalab, S.M., Unsteady MHD double-diffusive convection boundary layer flow past a radiative hot vertical surface in porous media in the presence of chemical reaction and heat sink, Meccanica, 48(2013), 931.

[16] Mosa, M.F., Mnaa, S.S., Abdulla, N.N., Thermal radiation effect in Ekman layer on a porous plate, Int. J. of Engg. Fluid Mech., 4(1991), no. 4, 427-448. 
[17] Muralidharan, M., Muthucumaraswamy, R., Thermal radiation on linearly accelerated vertical plate with variable temperature and uniform mass flux, Indian Journal of Science and Technology, 3(2010), no. 4, 398-401.

[18] Muthucumaraswamy, R., Ganesan, P., Radiation effects on flow past an impulsively started infinite vertical plate with variable temperature, International Journal Applied Mechanical Eng., 8(2003), no. 1, 125-129.

[19] Na, T.Y., Chiou, J.P., Laminar natural convection over a frustum of a cone, Applied Scientific Research, 35(1979), 409-421.

[20] Patra, R.R., Das, S., Jana, R.N., Ghosh, S.K., Transient approach to radiative heat transfer free convection flow with ramped wall temperature, Journal of Applied Fluid Mechanics, 5(2012), 9-13.

[21] Pop, I., Watanabe T., Free convection with uniform suction or injection from vertical cone for constant wall flux, International Communication in Heat Mass Transfer, 19(1992), 275-283.

[22] Pullepu, B., Ekambavanan, K., Pop, I., Finite difference analysis of laminar free convection flow past a non isothermal vertical cone, Heat Mass Transfer, 44(2008), no. 5, 517-526.

[23] Raptics, A., Radiation viscoelastic flow, International Communication Heat Mass Transfer, 26(1999), 889-895.

[24] Sharma, P., Varshney, C.L., Thermal dispersion effect on MHD flow of dusty gas and dust particles through hexagonal channel, International Journal of Heat Mass Transfer, 46(2003), 2511-2514.

[25] Viskanta, R., Grosh R.J., Boundary layer in thermal radiation absorbing and emitting media, International Journal of Heat Mass Transfer, 5(1962), no. 9, 795-806.

\author{
Bapuji Pullepu \\ Department of Mathematics \\ "SRM Institute of Science and Technology", \\ Kattankulathur, Chennai - 603203 \\ e-mail: Corresponding author: bapujip@yahoo.com \\ Rayampettai Munisamy Kannan \\ Department of Mathematics \\ "SRM Institute of Science and Technology", \\ Kattankulathur, Chennai - 603203
}

